\title{
EDITORIAL
}

Open Access

\section{Guest editors' introduction}

CrossMark

\author{
Kazuaki Kojima ${ }^{1 *}$ and Tomoko Kojiri ${ }^{2}$
}

\author{
* Correspondence: \\ kojima@lt-lab.teikyo-u.ac.jp \\ 'Learning Technology Laboratory, \\ Teikyo University, 1-1 Toyosatodai, \\ Utsunomiya 320-8551, Japan \\ Full list of author information is \\ available at the end of the article
}

\section{Special issue "modeling, management, and generation of problems/questions in technology-enhanced learning"}

Solving problems/questions is one of the most indispensable and important elements in the teaching and learning process. Problems/questions ask for specific domain knowledge and their difficulties are moderated by given and/or un-given conditions. Providing appropriate problems/questions enables teachers to assess the mastery levels of their students appropriately. In addition, problem/question-centered learning, including learning by problem solving and generation, allows students to apply learned knowledge to various situations.

In order to accomplish the problem/question-centered adaptive teaching and learning, it is essential to prepare a large amount of problems/questions on domain knowledge. In addition, appropriate metadata should be attended so as to select questions/problems that are appropriate for students.

This special issue attempted to provide a good and timely opportunity to present and share the results and issues of investigations about "problems/questions" in RPTEL community. We cordially invited papers interested in further exploring the many facets and potential uses of "problems/questions" in education/learning from a theoretical, technological, computational, pedagogical, sociological, or administrational point of views.

The first paper by Kojima, Miwa, and Matsui, Experimental Study of Learning Support through Examples in Mathematical Problem Posing, discussed learning from examples in learning of mathematical problem posing, in which students generate their own and novel problems, and experimentally investigated their effects. It argued the importance of a skill to pose novel problems. When using mathematics to solve problems in everyday life, problem solvers must recognize and formulate problems by themselves because structured problems are not provided. Therefore, fostering student problem posing is important but novice students have difficulty and occasionally fail in composing mathematical structures when posing problems. The paper proposed learning activities from examples to improve problem posing by novice students. Three learning activities, learning by solving an example, learning by reproducing an example, and learning by evaluating an example, were studied and their effects on composition of mathematical structures were reported.

The second paper by Horiguchi, Tomoto, and Hirashima, A Framework of Generating Explanation for Conceptual Understanding Based on Semantics of Constraints, proposed a framework for indexing problems to systematically generate explanation to promote acquisition of an ability to construct models of target systems (conceptual understanding) behind the problems in the domain of physics. Conceptual understanding is one of the

(c) 2015 Kojima and Kojiri. This is an Open Access article distributed under the terms of the Creative Commons Attribution License (http://creativecommons.org/licenses/by/4.0), which permits unrestricted use, distribution, and reproduction in any medium, provided the original work is properly credited. 
most important goals in science education. However, it is difficult to achieve the goal through conventional problem practice in which students solve several problems arbitrarily sequenced. Although conceptual understanding requires knowledge such as conditions in application of a solution and physical meaning of the solution, such knowledge is implicit for students. The paper designed a framework to systematically provide explanation to promote conceptual understanding. It illustrated the method in the framework to index models of problems by using constraints as knowledge descriptions and demonstrated generation of explanation with the framework. It also reported an empirical study to confirm whether explanation generated by the framework promoted student conceptual understanding.

The third paper by Le and Pinkwart, Evaluation of a Question Generation Approach Using Semantic Web for Supporting Argumentation, proposed an approach to generate questions used for stimulating student active discussions. An ability to generate arguments is important in education of social and science domains. Questioning can be deployed to advance the student argumentation ability, and teacher-initiated questions might stimulate students. To pursue question generation for support of students in developing their argumentation ability, the paper investigated questions automatically generated with WordNet, a lexical database. It described the approach while illustrating question generation in a discussion topic about production of nuclear energy and empirically studied whether questions in topics of energy and economy generated by human experts and a computer system were understandable, relevant to given topics, and useful for supporting students. It then compared the human- and computer-generated questions and discussed the effectiveness and limitations.

The fourth paper by Noguchi, Kogure, Konishi, and Itoh, Practice Supporting System with Related Problem Set Generator Based on Targeted Educational Effects, suggested an intelligent tutoring system that generates sets of similar problems, each of which is related to a certain educational target and supports exercises with the problem sets. An exercise in a classroom uses a set of multiple similar problems solved with common knowledge. To provide an appropriate problem set, teachers must carefully choose problems related with essential knowledge but that is not easy. The paper described an approach to generate such problem sets in the domain of high school chemistry. It empirically classified problem sets collected from textbooks and designed methods to generate similar problems for each type of the sets by transforming a problem. It then implemented the intelligent tutoring system by extending a previous system that can solve problems and explain about them. It also experimentally evaluated problem sets generated by the system and preliminary confirm the educational effect of exercises using the system.

The fifth paper by Matsuda, Ogawa, Hirashima, and Taki, A Generating Technique and Knowledge Representation of Multiple-Answer Problems for Learning with Solving Knowledge, addressed a method to generate erroneous answers and explanations about the errors in multiple-answer problems. In generation of multiple-answer problems, it is important to provide answers including errors potentially made by students and explanation for helping the students in correcting the errors as well as correct answers. Preexisting research has approached automatic generation of such erroneous answers and explanations for them. In implementation of such automatic generation, it is necessary but difficult to describe knowledge for solving problems in each respective domain. 
The paper proposed a technique for generation of erroneous answers and explanations, including knowledge descriptions enabling teachers to easily express their knowledge. It described a function to generate a correct answer from information about a problem and incorrect answers by perturbing the problem solving process to derive the correct answer. Moreover, it described a function to update knowledge through dialogues that require a teacher to confirm generated problems. It also presented empirical evaluations of the functions.

The sixth paper by Mishra and Iyer, An Exploration of Problem Posing Based Activities as an Assessment Tool, and as an Instructional Strategy, demonstrated the potential of problem posing in two dimensions: an instructional strategy and an assessment tool. It reviewed related research that proved the usefulness of problem posing and argued that different problem-posing situations can be used for different purposes because the results depend on design of the situations. The paper implemented problem-posing-based activities and empirically analyzed questions generated by students. It first conducted field studies in which the students were asked to generate questions to address their knowledge deficit or construct new knowledge in classroom settings. It described strategies the students used in generation of the questions and knowledge types the students used in or they unfolded by the questions. The paper then conducted another field study to investigate how to assess learning of students with questions they generated and how the qualities of the questions related to learning gains measured in traditional assessment. According to these studies, it discussed the potential of problem posing in the two dimensions.

We would like to thank the authors who submitted to this special issue and our reviewers who provided fruitful comments and suggestions.

\section{Kazuaki KOJIMA and Tomoko KOJIRI}

\section{Guest Editors}

Author details

${ }^{1}$ Learning Technology Laboratory, Teikyo University, 1-1 Toyosatodai, Utsunomiya 320-8551, Japan. ${ }^{2}$ Faculty of Engineering Science, Kansai University, 3-3-35 Yamate-cho, Suita 564-8680, Japan.

Published online: 09 July 2015

\section{Submit your manuscript to a SpringerOpen ${ }^{\circ}$ journal and benefit from:}

- Convenient online submission

- Rigorous peer review

- Immediate publication on acceptance

- Open access: articles freely available online

- High visibility within the field

- Retaining the copyright to your article

Submit your next manuscript at $>$ springeropen.com 